\title{
Rodolfo Lenz y la reforma ortográfica chilena: ciencia, tradición y política del lenguaje
}

\author{
Valentina Cáceres* \\ Universidad de Chile, Chile \\ Dario Rojas ${ }^{* *}$ \\ Universidad de Chile, Chile
}

\begin{abstract}
Resumen
En el presente artículo analizamos, desde el enfoque de la glotopolítica, las ideas de Rodolfo Lenz sobre la ortografía de la lengua española en el contexto de la pugna entre los partidarios de la ortografía reformada chilena y los adeptos a la ortografía de la Real Academia Española, en la década de 1890. La figura de Lenz es especialmente relevante en este marco por ser la figura más prominente de un paradigma por esas fechas recientemente incrustado en el campo cultural chileno: la ciencia del lenguaje. Analizamos un corpus de textos circunscritos a la década de 1890, que tienen como tema central o secundario la ortografía y la reforma ortográfica. En síntesis, concluimos que la defensa que Lenz hace de la ortografía chilena reformada, junto con su crítica a la ortografía de la Real Academia Española, se ven motivadas primero por el cientificismo
\end{abstract}

* Para correspondencia, dirigirse a: Valentina Cáceres (valentina.caceres@ug.uchile.cl), U. de Chile, Facultad de Filosofía y Humanidades, Departamento de Lingüística. Av. Capitán Ignacio Carrera Pinto 1025, piso 3, Ñuñoa, Santiago.

** Para correspondencia, dirigirse a: Darío Rojas (darioroj@uchile.cl), U. de Chile, Facultad de Filosofía y Humanidades, Departamento de Lingüística. Av. Capitán Ignacio Carrera Pinto 1025, piso 3, Ñunoa, Santiago. 
positivista, que articula las políticas modernizadoras que el Estadonación chileno auspiciaba por esos años, junto con verse influidas por la conciencia de estar enfrentando un problema cultural en el que la tradición juega un papel central.

Palabras clave: glotopolítica, ortografía, reforma ortográfica, ideología lingüística, Rodolfo Lenz.

\title{
Rudolf Lenz and the Chilean orthographic Reform: SCIENCE, TRADITION AND THE POLITICS OF LANGUAGE
}

\begin{abstract}
In the present article we analyze, from the glottopolitical approach, the ideas of Rodolfo Lenz on the orthography of the Spanish language in the context of the struggle between the supporters of the Chilean reformed orthography and the adherents to the orthography of the Royal Spanish Academy, in the decade of 1890. Lenz is especially relevant in this context for being the most prominent figure of a paradigm by that time recently embedded in the Chilean cultural field: the science of language. We analyze a corpus of texts circumscribed to the 1890 s, whose central or secondary theme is spelling and orthographic reform. In summary, we conclude that Lenz's defense of the Chilean reformed orthography, together with his criticism of the Royal Spanish Academy's spelling, are motivated first by the positivist scientificism, which articulates the modernizing policies that the Chilean nation-state sponsored for those years, together with being influenced by the awareness of facing a cultural problem in which tradition plays a central role.
\end{abstract}

Keywords: glottopolitics, othography, orthographic reform, language ideology, Rudolf Lenz.

Recibido: $15 / 12 / 18$

Aceptado: 02/03/19 


\section{INTRODUCCIÓN ${ }^{1}$}

Sin temor de exagerar, puede afirmarse que Rodolfo Lenz es la figura fundamental para el establecimiento de la lingüística moderna en Chile. Llegado desde Alemania en 1890, contratado por el Gobierno chileno para contribuir a la modernización de la formación de profesores de lengua en el Instituto Pedagógico, se transformó en el principal vector de la instalación de un nuevo paradigma, una nueva manera de entender el estudio del lenguaje, que en Chile hasta entonces había tenido sobre todo un carácter normativista (distinguir las variantes "correctas" de las "incorrectas" y promover el uso de las primeras) y se había enfocado en el lenguaje de las élites, la variedad culta de la lengua castellana ( $c f$. Rojas 2015, 2017). Frente a esto, Lenz abrazaba la concepción descriptivista, positivista y cientificista de la escuela histórico-comparada de los neogramáticos, en cuyo seno se había formado en Alemania. Esto introduce un punto de fuga y una fractura que reconfigura la tradición de los estudios del lenguaje en Chile.

Aunque la figura de Lenz ha concitado no poca atención de parte de los interesados en la historia de la lingüística en Chile y Latinoamérica (Álvarez Martínez 1997; Bernaschina 2013, 2016; Chávez 2011; Ennis 2012, 2016; Escudero 1963; Gómez Asencio 2016; Knauer 1993; Knauer y Kaluza 1998; Martínez 1997; Payas 2015; Pavez 2015; Rojas 2011; Sánchez 2013; Soto 2016; Velleman 2008) ${ }^{2}$, su intervención en los debates acerca de la reforma ortográfica en Chile ha ocupado un lugar más bien marginal en la bibliografía. Contreras (1994) se ha propuesto caracterizar de forma global las ideas ortográficas de Lenz, pero el alcance de su estudio es más bien descriptivo y adolece, según nuestra percepción, de pasar por alto las aristas políticas de la postura de Lenz, asunto ineludible si se tiene en cuenta que el reformismo ortográfico es un problema en que se revela de forma descarnada la condición política del lenguaje y de la actividad de los lingüistas (Villa $\&$ Vosters 2015). En cambio, las breves páginas en que Pavez (2015: 146-137)

\footnotetext{
1 Este artículo se enmarca en los proyectos I+D FFI2016-76874-P (Gobierno de España) y FONDECYT Regular 1150127 (CONICYT, Gobierno de Chile). Agradecemos especialmente a René Zúñiga, encargado del Fondo Lenz de la Universidad Metropolitana de Ciencias de la Educación, por facilitarnos el acceso a los materiales inéditos de Lenz que conformaron parte del corpus de esta investigación.

2 Por solo nombrar algunas referencias recientes, a las que habrá que sumar los trabajos reunidos en el volumen colectivo Lenguas, culturas y naciones: estudios en torno a Rodolfo Lenz, editado por Darío Rojas y Juan Antonio Ennis, de próxima aparición.
} 
comenta la postura ortográfica de Lenz apuntan con acierto en dirección al sentido político de dicha postura, pero sin llegar a adentrarse en esta arista.

En consideración de lo que acabamos de señalar, en el presente artículo nos proponemos interpretar con mayor profundidad las ideas ortográficas de Lenz desde el enfoque de la glotopolítica, lo cual, de partida, supone entenderlas, más que como "ideas", como "ideología", es decir, como reflejo del posicionamiento político de Lenz en el campo cultural y científico chileno.

La glotopolítica (Arnoux y Del Valle 2010; Del Valle 2017), enfoque de las ciencias del lenguaje de carácter emergente y de creciente aceptación entre los estudiosos de la relación entre lenguaje y sociedad, presupone entender el lenguaje como una práctica social más que como mera "forma" gramatical, y consiste específicamente en el estudio de las circunstancias materiales e históricas, políticas en sentido amplio, que influyen en las intervenciones, públicas o privadas, institucionales o individuales, sobre el campo de la lengua y sus manifestaciones artísticas y culturales. Desde este enfoque, se analizan tanto las prácticas (el uso de ciertas variantes en ciertos contextos comunicativos) como los discursos acerca de la lengua (sean textos ensayísticos sobre el lenguaje, textos gramaticales o simples conversaciones acerca de la lengua entre dos individuos), identificando en ambos casos "sus fundamentos ideológicos, su naturaleza performativa y las estrategias a través de las cuales constituyen su posición en el campo del cual y para el cual surgieron". (Del Valle 2016)

Cuando se asume una mirada historicista, los procedimientos metodológicos de la glotopolítica conllevan un importante componente empírico basado en la noción de archivo, tal como la entiende Derrida, es decir, como un conjunto de textos cuyo(s) sentido(s) debe ser atribuido y activado por el investigador al ponerlos en relación con sus circunstancias históricas. Así, el estudio del archivo pasa fundamentalmente por procedimientos de análisis del discurso, que es definido por Arnoux (2006) como la construcción empíricamente fundada de interpretaciones a partir de textos, la reconstrucción de sentidos para estos, atribuyéndoselos mediante la articulación de saberes procedentes de las ciencias del lenguaje (ya que todo texto es primeramente producto lingüístico) con saberes procedentes de otras disciplinas (historia, sociología, etc.).

En el enfoque glotopolítico, las prácticas lingüísticas y discursos sobre la lengua (sean de especialistas o de legos; de hecho esta distinción se relativiza) se entienden en necesaria y estrecha relación con el contexto social en que ocurren, de modo que una investigación como la presente, transdisciplinar por naturaleza, recurre a constructos teóricos provenientes no solo de la lingüística, sino también de las ciencias sociales. Por ello es 
que, en primer lugar, y como en casi todos los estudios de corte glotopolítico, recurrimos a la noción teórica de ideología lingüística (Del Valle y MeirinhoGuede 2016), que pone énfasis en la motivación política y vinculación con intereses de clase que están detrás de las representaciones sociales sobre el lenguaje. Partiendo de la larga tradición de las ciencias sociales que ha tratado el concepto de ideología (cf. Larraín 2007-2010), así como de las aproximaciones de la antropología lingüística y la historiografía de las ideas lingüísticas, la glotopolítica propone entender las ideologías lingüísticas como "sistemas de ideas que articulan nociones del lenguaje, las lenguas, el habla y/o la comunicación con formaciones culturales, políticas y/o específicas". (Arnoux y Del Valle 2010: 6)

Nuestro estudio, además de adoptar de forma novedosa el enfoque glotopolítico para aproximarnos a las ideas ortográficas de Lenz, se diferencia del estudio de Contreras (1994) por basarse en un corpus textual que excede el canon de textos lenzianos sobre ortografía y que cubre específicamente los primeros años de la presencia del alemán en Chile (1891-1896), que es cuando el debate sobre la reforma ortográfica acababa de recobrar vitalidad. Así, en primer lugar, consideramos los escritos más conocidos de Lenz al respecto, reunidos en De la ortografía castellana (Lenz 1914). Este reúne, por un lado, "De la ortografía castellana", que forma parte de una memoria presentada por Lenz al Director del Instituto Pedagógico y publicada en Anales de la Universidad de Chile como anexo a las actas de la sesión del 2 de julio de 1894 del Consejo de Instrucción Pública; y por otro, "Observaciones sobre la ortografía castellana”, que había sido publicado por primera vez en el periódico santiaguino La Libertad Electoral a fines de 1891, y luego reproducido como anexo a las actas del 2 de julio de 1894 por el Consejo de Instrucción Pública. Además, en segundo lugar, hemos examinado algunos textos epistolares inéditos de Lenz o dirigidos a Lenz, conservados en forma manuscrita en el Fondo Lenz de la Universidad Metropolitana de Ciencias de la Educación, en los que se aborda de forma central o tangencial el problema de la reforma ortográfica. De autoría de Lenz, logramos rastrear un borrador de una carta dirigida a Miguel Luis Amunátegui Reyes, que, aunque no tiene fecha, podríamos datar en 1894, puesto que Lenz aclara que la escribe a propósito de haberle pedido Amunátegui su opinión sobre el problema tras habérselo discutido en el Consejo de Instrucción Pública. Amunátegui Reyes, desde dentro de la Academia Chilena, sería luego uno de los más fervientes defensores de la ortografía reformada (Rojas 2018). Entre las cartas dirigidas a Lenz, de las que podemos inferir los temas y posturas que articulaban su interacción con otros intelectuales, destaca un documento remitido en 1896 por Aniceto dos Reis Gonçalves Viana (1840-1914), destacado fonetista portugués y miembro de la comisión que 
llevó adelante la reforma ortográfica portuguesa de 1911, de espíritu afín a la reforma chilena (simplificadora y basada en la pronunciación). También hallamos cuatro cartas que le fueron enviadas por el abogado chileno Aníbal Echeverría y Reyes (1864-1938) entre 1894 y 1895, con quien compartía el espíritu reformista y en quien Lenz influyó de forma gravitante para sus propias obras lexicológicas (Rojas 2011). Finalmente, incluimos en el corpus cuatro cartas del escritor costumbrista chileno Daniel Barros Grez (18341904), datadas entre 1895 y 1896, con quien Lenz compartía el interés por las manifestaciones de la cultura popular.

Habiendo ya declarado nuestro objetivo y su justificación, explicado el enfoque teórico-metodológico de nuestro estudio (la glotopolítica) y detallado el corpus que nos sirve de base, a continuación profundizaremos en algunos antecedentes que servirán para sostener una interpretación glotopolítica de la postura de Lenz frente a la ortografía chilena reformada.

\section{ANTECEDENTES}

\subsection{El REFORMISMO ORTOGRÁFICO CHILENO A FINES DEL XIX}

El reformismo ortográfico en la cultura lingüística hispanohablante tiene una larga historia, que se remonta al menos al Renacimiento y encuentra un punto culminante (aunque no final) en el siglo XIX (Martínez Alcalde 2010). Dicho momento histórico es importante sobre todo porque representa la proyección transatlántica de los debates acerca de la ortografía en lengua española (Villa 2016). En lo que concierne a América, en las recientemente independizadas naciones hispanohablantes, la escritura y la alfabetización ocuparon un lugar principal entre las preocupaciones de los forjadores de los Estados-naciones modernos, debido al rol que la palabra escrita desempeñaba en la circulación de las ideas, la comunicación internacional y, especialmente, el conocimiento y observancia de la ley (Jaksic 1999).

De tal modo, en Chile, durante las primeras décadas de la Independencia, surgieron iniciativas de reforma ortográfica que buscaban simplificar y racionalizar la escritura con el fin de facilitar la alfabetización de la población (Contreras 1993). Una de las primeras intervenciones, en este sentido, fue la de Juan García del Río y Andrés Bello ([1823] 2013), publicada durante el exilio londinense de estos intelectuales americanos. A diferencia de la ortografía de la RAE, esta propuesta se inclinaba por el criterio fonético 
en desmedro de los criterios etimológico y usual. Años más tarde, en 1843, cuando ya Andrés Bello era rector de la recién fundada Universidad de Chile, se le encargó al argentino Domingo Faustino Sarmiento (por entonces exiliado en Chile) escribir una Memoria sobre ortografía americana, publicada en 1843, que añadía al fonetismo un acentuado americanismo (la ortografía debía reflejar la pronunciación corriente entre los americanos).

En 1844, la Universidad de Chile tomó la decisión de promover a nivel oficial en el país una ortografía que se alimentaba eclécticamente y con gran moderación de las propuestas anteriores, y que de hecho rescataba sobre todo las propuestas de García del Río y Bello, al tiempo que rechazaba las sugerencias más radicales de Sarmiento. Esta ortografía, que se conoció como "chilena" o "casera", se diferenciaba de la de la RAE en tres puntos fundamentales: el uso exclusivo de $<\mathrm{j}>$ para el sonido $/ \mathrm{x} /$ (jente), el uso exclusivo de $<\mathrm{i}>$ para /i/ con valor vocálico o semivocálico (soi, lei), reservando la $<\mathrm{y}>$ para el sonido consonántico; y finalmente, el uso de $<_{\mathrm{s}}>$ en lugar de $<\mathrm{x}>$ en grupos consonánticos como los de estraño. Sin embargo, tras un breve periodo de vigencia oficial, en 1851 el propio Bello "recomendó la cancelación de la reforma y el Ministerio de Educación Pública impuso un decreto a tal efecto" (Velleman 2004: 48-49). A pesar de ello, la ortografía chilena siguió usándose ampliamente en textos impresos de circulación pública hasta 1927.

En las décadas inmediatamente anteriores al momento en que Lenz llega a Chile (1890), las discusiones sobre ortografía habían cejado en cantidad e intensidad, en comparación con lo acontecido entre 1830 y 1850 . En las dos últimas décadas del XIX, sin embargo, ocurrieron varios acontecimientos que reactivaron el interés por la reforma ortográfica. Primero, en 1884 el Ministerio de Instrucción Pública consultó al Consejo Superior de Instrucción Pública acerca de la conveniencia de reimprimir para uso de las escuelas chilenas ejemplares de la Ortografía de la Real Academia Española, lo cual significaría un espaldarazo a la ortografía académica; tras largas discusiones, dilatadas hasta 1888 , en que también participaron integrantes de la Facultad de Humanidades de la U. de Chile y miembros chilenos correspondientes de la RAE, se acordó recomendar (sin efecto considerable) la adopción de la ortografía académica.

Segundo, en 1885, por iniciativa de la Real Academia Española, se había fundado la Academia Chilena correspondiente de la Española, entre cuyos propósitos fundamentales se encontraba precisamente abogar por la ortografía académica en aras de la unidad idiomática (Rojas 2016). Si bien en el periodo fundacional (1885-1888) la campaña pro ortografía académica se limitó sobre todo a declaración de propósitos, en la época de reinstalación de la Academia Chilena, iniciada en 1914, sus miembros 
tomaron una disposición de activo compromiso con su causa (Gutiérrez 2017; Rojas 2018). Manuel Salas Lavaqui, secretario de la corporación desde 1914, hizo un fuerte lobby para que la Facultad de Humanidades de la U. de Chile aprobara su proposición de que se adoptara la ortografía académica en la enseñanza. Asimismo, Salas Lavaqui gestionó y logró que un grupo de senadores (Manuel Salinas, Carlos Aldunate Solar, Eliodoro Yáñez y Francisco Valdés Vergara) presentara al Senado una propuesta de ley en este mismo sentido. Pese a la oposición que desde dentro de la Academia Chilena presentó con mucha resonancia Miguel Luis Amunátegui Reyes, quien defendía a rajatabla la ortografía chilena, las discusiones acabaron en 1927, cuando mediante un decreto del presidente Carlos Ibáñez del Campo se determinó el abandono de la ortografía chilena en el ámbito oficial y su sustitución por la ortografía de la RAE.

En tercer lugar, de manera casi simultánea a la intervención de Lenz en asuntos ortográficos, y posiblemente motivados por los dos elementos contextuales mencionados anteriormente, en 1892 emerge a la luz pública el movimiento de los neógrafos (Payas 2008; Villarroel 2017). Estos, encabezados por Carlos Cabezón, adoptaron una actitud de reformismo radical, fundamentada en el positivismo cientificista y en ideas libertarias (anarquistas), y materializada a través de una ortografía estrictamente basada en la pronunciación, llamada por ellos ortografía rrazional. Llevaron a la práctica sus ideas mediante la publicación autofinanciada de traducciones y de obras propias impresas en este sistema ortográfico. Sin embargo, sus ideas, más allá de generar una reacción negativa entre los sectores más conservadores (como la Academia Chilena), no lograron influir en las prácticas ortográficas de los hispanohablantes en Chile.

A manera de síntesis, entonces, al momento de intervenir Lenz en las polémicas ortográficas chilenas, estas, luego de haber estado en un estado de latencia desde la década de 1850 , empezaban a cobrar fuerzas otra vez gracias a la aparición de nuevos actores glotopolíticos y elementos contextuales. Estos actores representaban, por un lado, una tendencia culturalmente conservadora e hispanista, a favor de la ortografía de la Real Academia Española, y otra partidaria de radicalizar los principios que fundamentaron la ortografía chilena reformada, todavía en vigencia pero cada vez más presionada por el asedio de los sectores hispanistas.

\subsection{LenZ y la introducción de la “Ciencia del lenguaJe” en Chile}

El contexto de fines del siglo XIX en Chile está marcado por un acelerado proceso de modernización de la sociedad chilena -y latinoamericana en 
general-, que está ligado "a la expansión mundial del mercado capitalista" (Subercaseaux 2011:348). Este proceso modernizador repercute directamente sobre las diversas instituciones, la economía y los modelos culturales y sociales que se tenían como referencia durante la época. Por ello, dentro del plano social, la educación se convirtió en la preocupación fundamental de intelectuales como Valentín Letelier, Claudio Matte, José Victorino Lastarria, Miguel Luis Amunátegui, entre otros. Principalmente gracias a los esfuerzos de Letelier, en abril de 1899 se dictó el decreto de creación del Instituto Pedagógico, articulado sobre la necesidad de formar nuevos profesores y sobre la contratación de docentes alemanes para dicha institución. Dentro de estos, destacan las figuras de Rodolfo Lenz, Federico Hanssen, Federico Johow, Augusto Tefelmacker, entre otros, quienes venían a impartir clases de humanidades -subdivididas en: historia y geografía, castellano y latín, inglés y alemán, griego y francés- $\mathrm{y}$ de ciencias -subdivididas en ciencias naturales y matemáticas- (Sanhueza 2010). Resulta importante señalar que lo fundamental del Instituto Pedagógico fue la idea de formar con criterios científicos, ya que se creía en la necesidad de una ciencia de la educación, esto entendido en el afán estatal de la época por modernizarse.

Rodolfo Lenz llega a Chile en 1890 con la misión primera de impartir docencia superior destinada a la formación de profesores de idiomas modernos (francés, inglés e italiano) y, posteriormente, asume tanto la cátedra de gramática española como la de gramática histórica castellana. Introdujo una importante fractura en las tradiciones del estudio del lenguaje en Chile, pues, frente al normativismo y la predilección por el estudio de las variedades cultas y literarias del castellano, tendencia típica del siglo XIX chileno (Rojas 2017), Lenz consideró en cambio del mayor interés para la lingüística moderna el estudio del "dialecto vulgar" del castellano chileno y de lenguas indígenas como el mapudungun. Debe tenerse en cuenta, para entender la postura de Lenz, que, hacia fines del siglo XIX, la lingüística, de cuño positivista pero todavía historicista, había dejado de interesarse en la lengua literaria y declaró que la verdadera naturaleza del cambio lingüístico solo podría conocerse a través del estudio de las variedades populares y del lenguaje vivo en boca del pueblo, el que experimentaba en esos precisos momentos el cambio y el dinamismo inherentes al lenguaje (Swiggers 2011). Asimismo, a partir de la época de August Schleicher (1821-1868), la disciplina se había preocupado sobremanera de ganar el estatus de ciencia, a partir del modelo de las ciencias naturales (Ennis 2014).

En consecuencia, la actitud cientificista y descriptivista de Lenz frente al lenguaje, novedosa para el contexto chileno, se enfrentó a una importante resistencia por parte del campo cultural chileno, que básicamente todavía seguía pensando con Bello que la gramática era el "arte de hablar y escribir 
correctamente". De esto da cuenta por ejemplo el ácido cuestionamiento de Eduardo de la Barra hacia los profesores alemanes del Pedagógico (Velleman 2007) o la reseña crítica del sacerdote e hispanista, miembro de la Academia Chilena, Manuel Antonio Román a su Diccionario etimológico (Lenz, s.f.). Precisamente en la crítica de Román al diccionario de Lenz encontramos el contraste expresado de manera diáfana:

Hasta ahora todos habíamos creido que la enseñanza de la gramática era para hablar y escribir correctamente, como la gente educada; pero ahora el Dr. Lenz nos dice que estábamos profundamente equivocados y que la gramática, por lo menos en Chile, es para hablar y escribir 'la lengua huasa'. (cit. en Lenz, s.f.)

Siempre hemos tenido un alto concepto de la ciencia lingüística y filológica, como que es el estudio de uno de los dones mas grandes que el Criador ha otorgado a su criatura, cual es el lenguaje, y estudio, al mismo tiempo, del pensamiento humano en todas sus manifestaciones por medio de la palabra hablada y de la escrita. Por esta razón hemos creído y creemos que todo el que se dedique al estudio del lenguaje tiene la noble misión de encauzarlo en las leyes de la gramática, desbastándolo, puliéndolo y limpiándolo según las exigencias de la civilización y de la simple educación. Si así no fuera, la ciencia del lenguaje no sería ciencia sino mero inventario de palabras, desde la que estropea con su mala pronunciación el último patán, hasta las que profieren con cínica desvergüenza el borracho en la taberna y el frecuentador de burdeles. (cit. en Lenz, s.f.)

Lenz contesta a Román de la siguiente manera, revelando los horizontes completamente distintos que tenían, por un lado, los especialistas científicos de la época (representados por Lenz) y, por el otro, los cultores del normativismo (representados por Román):

Esta definición del estudio del lenguaje (digamos de la lingüística) es tan correcta como si se dijera que la botánica tiene por objeto producir hermosas flores, peras dulces y papas grandes. La confusion entre retórica y lingüística es a lo menos falta tan grave como si se declarara que horticultura y botánica son una misma cosa. La mala pronunciación del último patan puede tener para la lingüística el mismo interés que un cultivo de bacilos de la peste bubónica para la biología y la medicina. (Lenz, s.f.).

En síntesis, nuestra interpretación de las ideas ortográficas de Lenz requerirá considerar, además de lo que atañe específicamente al reformismo ortográfico, cómo las claves contextuales de la modernización del Estado- 
nación chileno y de la introducción de una "ciencia del lenguaje" en el país, en pugna con la tradición hegemónica normativista, de inspiración bellista, influyen en la posición que adopta el lingüista alemán frente al reformismo ortográfico chileno.

\section{LENZ Y EL REFORMISMO ORTOGRÁFICO CHILENO}

\subsection{LENZ: PARTIDARIO DE LA ORTOGRAFÍA REFORMADA}

En el material textual que analizamos, Lenz, sin ninguna clase de ambages, se declara partidario de la ortografía reformada chilena y se muestra abiertamente crítico de la posibilidad, latente por mediados de la década de 1890, de que el Estado chileno adoptara la ortografía de la Real Academia Española:

[...] la ortografía chilena es mucho mas científica, lójica i fácil que la de la Real Academia Española. La pedagogía debe en todo caso preferir la ortografía chilena, porque es sumamente importante hacer tan fácil como sea posible el aprendizaje de la lectura i escritura correcta. La ortografía académica $[\ldots]$ no tiene ninguna ventaja sobre la de Bello, pero sí muchas desventajas. No veo, pues, ninguna razon para abandonar el buen uso jeneral de Chile en favor del malo de España. (Lenz 1894: 38)

No ofrece una propuesta concreta de norma ortográfica ni un modelo para seguir en la práctica, como hicieron muchos otros intervinientes en estas polémicas (Contreras 1993): se contenta más bien con tomar partido en la pugna existente entre reformistas y contrarreformistas y expone las razones que tiene para apoyar a los primeros.

En la toma de postura de Lenz hay una estrategia de autorrepresentación como "observador externo" frente a la discusión, la que saca a colación en el borrador de una carta (c. 1894) dirigida a Miguel Luis Amunátegui Reyes:

Con motivo de haberse tratado en el seno del Consejo de instrucción pública de la cuestión ortográfica usted tuvo a bien pedir mi opinión sobre el particular. Habiéndome interesado por las cuestiones ortográficas desde mi llegada a Chile, he estudiado los artículos i folletos que se han publicado sobre este asunto importante i he llegado a armarme una idea fija sobre esta cuestión que creo poder defender contra todo ataque que se le pudiera hacer, i que quizás tendrá algún interés para usted puesto que 
en mi cualidad de estranjero creo ser absolutamente imparcial. No me pueden mover el orgullo nacional que tantas veces suele ofuscar el juicio de los hombres, porque no soi parte en la causa, sino sacaré las razones para mi decisión únicamente de los conocimientos i las esperiencias que creo haber adquirido por mis estudios lingüísticos i fonéticos i por mi ocupación en la enseñanza de idiomas maternos.

Esta idea se reitera en la nota al lector incluida en la reedición de 1914 de sus escritos sobre ortografía publicados originalmente en 1891 y 1894: "Siendo mi esposicion fundada en hechos $i$ apreciaciones cientificas que son hoi lo que fueron hace casi veinte años, no hai necesidad de introducir cambios mayores en la redaccion" (Lenz 1914: 11; resalte nuestro). Igualmente, es mencionada en la introducción de su primer trabajo publicado sobre el particular:

Para poder decidir cuál de las dos ortografías es la mejor, i por esto preferible a la otra, tenemos que entrar, sine ira et studio, en un lijero exámen de la historia de la ortografía castellana i de las bases razonables $i$ aprobadas por la ciencia de nuestros dias sobre las cuales se debe fundar teóricamente toda reforma ortográfica que quiera ser juzgada buena y provechosa. (Lenz [1891] 1894a: 42; resaltes nuestros)

De esta forma, Lenz se construye a sí mismo como alguien cuya opinión está revestida de objetividad y libre de subjetividades nacionalistas que pudieran ofuscar la acción de la razón, condición necesaria para la intervención de un sujeto glotopolítico cuya autoridad está sustentada en su condición de científico.

El ideologema de la razón adquiere su importancia, creemos, gracias a la relevancia que este concepto tenía para el principal interlocutor de Lenz, el aparato gubernamental chileno, en el contexto de modernización, sobre bases cientificistas y positivistas, en que se sitúa la discusión. Tomar una decisión a partir de lo razonable, conduciría inevitablemente al progreso, ideal anhelado por los gobernantes chilenos desde la fundación de la nación. Por el contrario, desconocer el dictado de la razón conduciría a un retroceso. En una de las cartas que conservó Lenz de su correspondencia con Viana aparece claramente esta idea, que, reafirmada por una autoridad como era el fonetista portugués, con mayor razón sostendría Lenz: "Algunas palabras me permitirá sobre la ortografía chilena. A mi me parece que es un perfeccionamiento de la Academia, i que sería una calamidad el retroceder". (Viana 1896)

En consecuencia, Lenz apela al "buen juicio" de las elites gobernantes en cuanto a las decisiones lingüísticas que debían ser tomadas en materias 
ortográficas ("Unicamente la fuerza brutal puede obligar a un hombre de buen juicio a aceptar lo malo por lo bueno"; [1891] 1894a: 51), contrastándolo con el "juicio estraviado" de la RAE ([1891] 1894a: 54), al tiempo que se preocupa de declarar inevitable, por lo mismo, el triunfo de la postura reformista ("La razon vencerá, i ella está en el camino...", 1894b: 18; "Tan seguro como es el progreso continuo de la humanidad, tan seguro es que, tarde o temprano, los otros pueblos españoles i España misma adoptarán la ortografía mas razonable que nació en Chile", 1894b: 39-40). La manifestación más hiperbólica de la importancia de la razón (científica) en estas materias es la siguiente afirmación: "Esta ortografía americana, es, pues, un progreso en el camino de la reforma ortográfica; seria casi un suicidio de la razon si diéramos un paso atras" (1894b: 51; resalte nuestro).

En consecuencia, el primero de los fundamentos de su postura será el cientificismo, que en relación con la ortografía toma la forma de un argumento respaldado en la fonética, por entonces emergente disciplina de las también recientemente institucionalizadas ciencias del lenguaje. Si este eje de sentido de los discursos de Lenz tiene una naturaleza "ahistórica" (en el sentido de 'universal y atemporalmente válida'), no quiere decir que Lenz desconozca que el problema de la reforma ortográfica era histórico, en el sentido de que estaba inmerso en una serie de prácticas sociolingüísticas y culturales cargadas de indicialidades (según la terminología de la antropología lingüística; $c f$. Silverstein 2003) y propias de una comunidad hablante/escribiente concreta. Por esto es que el segundo fundamento de la postura de Lenz alude a cómo esta dialoga con la tradición, punto en que esencialmente se dedica a discutir acerca de la legitimidad de la Real Academia Española y los fundamentos de su ortografía y del lugar de la reforma en la historia cultural de Chile.

En lo que sigue, revisaremos en detalle estos dos grandes ejes de sentido (3.2 y 3.3, respectivamente) que atraviesan los discursos de Lenz que forman parte de nuestro corpus.

\subsection{LA CIENCIA DEL LENGUAJE Y LA ORTOGRAFÍA}

Para Lenz, la ortografía debe basarse en los criterios científicos de la disciplina por entonces hace relativamente poco (comienzos del XIX) denominada "lingüística" (Swiggers 2011). Desde el punto de vista de esta disciplina, en primer lugar, la ortografía ideal debe atenerse a la pronunciación, en congruencia con la idea de que las lenguas son primero que nada manifestaciones orales, establecida como axioma por el paradigma lingüístico hegemónico del último cuarto del siglo XIX, en el 
cual precisamente se formó Lenz (la lingüística histórico-comparada de los neogramáticos). En el texto inicial de la serie que compone nuestro corpus, Lenz establece las bases lingüísticas de su razonamiento:

1. ${ }^{\circ}$ Teniendo toda escritura por único i exclusivo fin el representar al conocimiento de cada cual por medio de la vista, palabras i frases de la lengua que en primer lugar es lengua hablada, es evidente que, teóricamente, la escritura es tanto mejor cuanto mas exactamente represente la pronunciacion [...].

$2 .{ }^{\circ}$ No se debe ni se puede exijir mas de la escritura; especialmente, por regla general, será inútil e innecesario que la escritura distinga palabras que no esten separadas en la pronunciacion. [...] Aumentar las dificultades ineludibles de la ortografía con otras inventadas caprichosamente, es robar al pueblo entero muchas horas que mejor se emplearian en la enseñanza de otros ramos útiles.

$3{ }^{\circ}$ Con esto queda incontestablemente establecido que la ortografía ideal tiene que ser una ortografía fonética, en la cual a cada sonido corresponda un solo signo gráfico i a cada signo gráfico un solo sonido pronunciado [...; resalte en el original].

4. ${ }^{\circ}$ Como la lengua hablada se desarrolla lenta, pero irresistiblemente, una vez establecida una ortografía mas o menos perfecta, ella no puede quedar inalterable [...]. Por esto, aun la mejor ortografía deberia ser alterada de vez en cuando [...]. (Lenz [1891] 1894a: 43-44)

Los tres primeros puntos establecen claramente el carácter "fonético" de la ortografía ideal de Lenz. Aunque usa el término "ortografía fonética", cabe tener en cuenta que la ortografía ideal para él se caracteriza por ser lo que hoy llamaríamos fonémica ${ }^{3}$, pues, como detalla en el primer punto, cuando establece la "pronunciación" como punto de referencia se refiere "no [a] la pronunciacion individual de un solo hombre, sino aquel cánon de pronunciacion que se forma por la abstraccion de todas las peculiaridades individuales que no coinciden con el término medio usado entre los hombres instruidos de una comunidad lingüística" (Lenz [1891] 1894a: 43). Este "cánon de pronunciacion", por otra parte, corresponde al habla de las personas cultas, y no al de los sectores populares sin educación formal. En un espíritu sorprendentemente afín al de la postura de Bello y sus epígonos

\footnotetext{
3 Aunque la idea del fonema está presente en los estudiosos del lenguaje desde hace varios siglos, el término phoneme, acuñado por Dufriche-Desgenettes en 1873 y usado por Saussure desde 1878, fue desarrollado en profundidad por Baudouin de Courtenay y Kruszewski (Van der Hulst 2013). Lenz, hasta donde sabemos, no hizo una adopción temprana de este término.
} 
(volveremos luego sobre esto), Lenz advierte: "No haya temor de que se propongan como modelo pronunciaciones que han de tomarse por viciosas, porque no son reconocidas de la mayoría de los hombres cultos que hablan el castellano" ([1891] 1894a: 54).

En segundo lugar, el cuarto punto desarrollado por Lenz se desprende del historicismo propio de la lingüística europea del siglo XIX, que hay que entender en los términos establecidos por Hermann Paul (el principal teórico de los neogramáticos; $c f$. Koerner 2008): solo una aproximación historicista al estudio del lenguaje es verdaderamente científica. En consecuencia, si se toma como axioma la naturaleza cambiante y dinámica de las lenguas en el tiempo, resulta que la pronunciación, incluso entre los sujetos cultos, está sujeta a transformación histórica $\mathrm{y}$, por ende, la ortografía requiere obligatoriamente ajustes con el paso del tiempo. Precisamente este es, para Lenz, el problema fundamental de la ortografía de la RAE: corresponde a un estado anterior de la historia de la pronunciación castellana. Al mismo tiempo, es la justificación para que se haya reformado la ortografía en Chile, reparando así el desajuste histórico de la ortografía académica. De esta manera, la ortografía chilena reformada también es más racional que la de la RAE porque responde al hecho científico de que el lenguaje (oral) cambia con el tiempo.

La importancia de que una opinión sobre la ortografía en Chile se fundamente en bases científicas aparece también recurrentemente en las cartas que Lenz recibió entre 1895 y 1896 del escritor Daniel Barros Grez.

Por grande que sea el respeto debido [...], mayor debe ser siempre el que hemos de profesarle a la ciencia. (Barros Grez 1896(a))

[...] al fin la opinión pública, en jeneral, ha de reaccionar en favor de la ciencia. (Barros Grez 1896(b))

Pero, aunque no soi un fonetista, amo tan de veras la ciencia, esto es, la verdad de las cosas. (Barros Grez 1896(c))

Junto con lo que hemos destacado en los párrafos precedentes, la vertiente cientificista de los argumentos de Lenz conduce a la activación de argumentos de autoridad que remiten al grupo profesional con el que Lenz se identifica y sobre la base del cual construye su propia legitimidad: "Los lingüistas de todo el mundo dan preferencia a las ortografías fonéticas" ([1891] 1894a: 52). Junto con esta mención en general a los lingüistas, ya establecidos como "científicos" en la disciplina a fines del XIX, Lenz cita a algunas figuras clave de la lingüística científica contemporánea, específicamente de los estudios fonéticos, para respaldar su postura. Entre ellos destaca Fernando de Araujo, fonetista español, autor de Estudios de fonética castellana (1894), obra de amplia repercusión internacional en su época (Martínez Celdrán y Romera 
Barrios 2007: 128), que definía su disciplina precisamente como "ciencia psíquico-acústica-fisiológica" y destacaba su condición "modernísima" (cit. en Muñiz Cachón 2009: 484), características ambas que coinciden con aquello que probablemente le interesaba destacar a Lenz en función de persuadir al aparato estatal chileno. Lenz califica a Araujo como "el español hoi en dia mas competente en estas materias" (1894b). Debe tenerse en cuenta que tanto Araujo como Lenz escriben por los años en que había aparecido la Asociación Fonética Internacional (AFI, fundada en 1886), liderada por el francés Paul Passy, la que logró institucionalizar la cientifización del estudio de los sonidos del habla y que precisamente se pensaba como una base para la ortografía y la enseñanza. Cabe destacar que Lenz fue un participante muy activo en dicha asociación, que mantuvo correspondencia con Passy, y que, gracias a su gestión, Chile fue uno de los países más representado en la AFI durante sus primeras décadas de existencia (por 1914, había 83 miembros chilenos, frente a los 10 de España, por ejemplo; Ortiz Lira 1994: 5).

Cabe preguntarse: ¿por qué Lenz cita a Araujo, y no a otros fonetistas europeos? La explicación más plausible es la de que se trataba de un fonetista de habla castellana, que escribió sobre la posibilidad de reformar la ortografía castellana, y que, por lo mismo, primero, era una figura relevante para la discusión particular de la que se ocupaba Lenz, y,segundo, el hecho mismo de ser español y no americano, servía para reforzar la idea, que veremos en el apartado siguiente, de que incluso entre los mismos españoles había quienes se daban cuenta de la falta de idoneidad de la ortografía de la RAE (y que por tanto no era una cuestión meramente de patriotismo). Adicionalmente, Araujo formaba parte de las redes intelectuales consideradas legítimas por Lenz: sus Estudios de fonética castellana reunían una serie de trabajos publicados originalmente en la revista Phonetische Studien, dirigida por Wilhelm Vietor en Leipzig, capital del movimiento neogramático.

\subsection{LA REFORMA ORTOGRÁFICA COMO PROBLEMA CULTURAL: LA TRADICIÓN}

\subsubsection{La ortografía de la Real Academia Española}

Recordemos que la pugna ortográfica, en la última década del XIX, enfrentó a los partidarios de la ortografía casera o chilena con los partidarios de la ortografía de la RAE, y que Lenz se encontraba entre el primero de estos bandos. En consecuencia, junto con valorar positivamente la ortografía reformada por su carácter afín a los dictados de la ciencia lingüística, como vimos en el apartado anterior, los textos de Lenz se encargan de destacar los problemas de la ortografía académica, entre los cuales sobresale el problema de su apego a la etimología, en lugar de limitarse a la pronunciación. 
Sin embargo, Lenz plantea esta crítica de una manera estratégica, pues sabe que la ortografía académica gozaba de apoyo entre sectores conservadores muy poderosos y cercanos a las altas esferas del Gobierno, y que la ideología lingüística hegemónica de la élite hispanohablante chilena tenía una inclinación a veces casi reverenciosa por la Real Academia Española y sus normas. Por esto, un defensor de la ortografía reformada de alguna manera iba a contracorriente, sobre todo tras varias décadas de lo que algunos llamaban un "caos ortográfico" (Contreras 1993) y del que se culpaba sobre todo a los reformistas. En suma, Lenz debe plantear su opinión sin olvidar que se enfrenta a una tradición de ideas y posturas que no puede simplemente descalificar sin más.

En primer lugar, hay una serie de embistes recurrentes de Lenz a la Real Academia Española que son en realidad una derivación directa del criterio cientificista, pues su fundamento es el cuestionamiento de las credenciales científicas de la corporación madrileña, desde el punto de vista de la "ciencia moderna", lo que explicaría la naturaleza poco científica de su ortografía:

[...] esta autoridad es nula, si no está fundada en la incontestable superioridad de conocimientos, en la absoluta competencia de 1 os miembros. Pero la Real Academia Española no es de ninguna manera infalible, i, sin disminuir el gran mérito literario que pueda tener, debemos confesar que en materia de ortografía, como en materias lingüísticas, en jeneral, los actuales miembros de dicha Sociedad no nos parecen estar a la altura de la ciencia moderna: bastarian para probarlo 1as numerosas etimolojías incorrectas e incontestablemente falsas de la última edicion del Diccionario. (Lenz [1891] 1894a: 52)

$[\ldots]$ pues se sabe que los resultados de los trabajos oficiales no dependen esclusivamente de los mas competentes miembros sino de todos los Académicos a la vez, i ¿quién puede negar que entre los $<<$ Individuos de número $>>$, en la elección de los cuales entran tantas consideraciones políticas i sociales, hai mas de un número cero que debe su pertenencia a la Academia tan solo a sus títulos de nobleza i a sus méritos políticos? [...] Ahora, en vano me pregunto por qué tendrá una posicion escepcional la Real Academia Española, a no ser que todas sus obras revistan un carácter cientifico de competencia irreprochable. Pues bien: por desgracia, es sumamente fácil probar que la Academia no tiene ni sombra de competencia en materias lingüísticas. (Lenz 1894b: 21-22)

[...] el Diccionario de la Real Academia Española trae millares de disparates, que hubieran sido perdonables en el siglo XVIII, pero que hoi prueban la absoluta falta de 1 os mas elementales conocimientos lingüísticos. (Lenz 1894b: 23) 
En la segunda de estas citas, puede apreciarse además la acusación de que los miembros de esta corporación suelen formar parte de ella por méritos políticos o capital social, más que por su condición de especialistas.

Además de esta crítica cientificista a la RAE, Lenz lleva el cuestionamiento al terreno de lo geopolítico, pues considera que la institución madrileña no debe ser considerada autoridad idiomática en Chile precisamente por ser una entidad española. Que el gobierno chileno tome una decisión a partir de la autoridad de una institución española es, para Lenz, contradictorio con la emancipación de las naciones americanas:

La autoridad qua asume la Real Academia Española no tiene ninguna importancia para los americanos. (Lenz [1891] 1894a: 52)

Las indagaciones i opiniones de la Real Academia Española no tienen, segun nuestro parecer, ningun derecho propio y particular que no lo tengan las demas Academias. (Lenz 1894b: 20)

No debe perderse de vista que Lenz fue contratado por el Gobierno chileno y que su labor en el Instituto Pedagógico y en los campos científico y cultural locales respondían a intereses gubernamentales, los que buscaban consolidar el sistema educativo que se estaba forjando con la finalidad de fomentar un ideal de ciudadano republicano que cultivara la lealtad al nuevo Estadonación (Jaksic 1999: 509). No es raro, entonces, que, a pesar su declarada posición de observador externo, atraiga a la discusión una dimensión patriótica, pero que tiene como sujetos a las entidades chilenas involucradas, y no a él como mero "informante". En resumen: el Estado chileno debería promover la ortografía reformada, ente otras razones, porque es chilena, y no la de la RAE, porque es española.

La falta de autoridad de España en Chile, por otro lado, aparece vinculada con el argumento anterior de la falta de cientificidad de la RAE. No se trata solo de la RAE: es la nación española la que está a la zaga de la ciencia moderna, según Lenz, y por eso es que las respuestas (científicas) a los problemas de las naciones americanas independientes hay que buscarlas en los países desarrollados de Europa, entre los que no se cuenta España, pero sí, por ejemplo, Alemania y Francia:

I ¿quién se atrevería a sostener que las lucubraciones de la Real Academia de Medicina son de autoridad absoluta i obligatoria para el Cuerpo Médico de Chile, cuando sabemos que 1os médicos americanos que quieren perfeccionar sus estudios en Europa van a Alemania i a Francia, pero no a España? (Lenz 1894b: 21) 
Lenz se encarga, en particular, de recordar que Alemania, de donde él mismo proviene y a donde acudieron los creadores del Instituto Pedagógico para cientifizar y modernizar la enseñanza en Chile, es el centro de las ciencias del lenguaje en ese momento, y que las teorías y métodos desarrollados en aquel país han sido exportados a muchas otras partes con mucho éxito (excepto a España):

Es un hecho por demas conocido i lastimoso que la semilla que sembró el sabio fundador de la lingüística neolatina Federico Diez, profesor de la Universidad de Bonn en Alemania, en todos los países de la raza latina ha brotado i ya ha dado espléndidos frutos, ménos en España. (Lenz 1894b: 22)

Una excepción que Lenz podría haber concedido es la de Fernando Araujo, fonetista español al que, como vimos en 3.2, atribuía la condición de verdadero experto; pero si la tenía, era no por representar lo corriente en España, sino más bien por estar vinculado con las capitales europeas de la ciencia del lenguaje.

Otra nación europea a la que Lenz podría haber propuesto mirar como modelo era Portugal, que en 1911 haría oficial una reforma ortográfica de espíritu similar a la reforma chilena, basada en las propuestas que por la última década del XIX habían hecho Aniceto dos Reis Gonçalves Viana y otros. En la correspondencia de Lenz con Viana, destacado fonetista portugués, en 1896 este le comunicaba (como ya vimos) su parecer respecto de la idoneidad de la ortografía reformada chilena. En otra carta, enviada por el portugués en 1911, año de oficialización de la reforma, le indica a Lenz:

[...] las simplificaciones que hace tanto tiempo yo venia defendiendo y propagando han sido adoptadas por la Comisión de la que yo hacía parte también, nombrada por el Govierno. Tenemos enfín nosotros los portugueses una ortografía en lo posible, tan sencilla y regular, si no mas, que la castellana y la toscana. Hay en el público de literatos [ilegible] alguna oposición, como siempre la hubo en todas partes, contra la simplificación: se dice, pero sin eco favorable, que así se deturpa la lengua, y que por patriotismo(!) debiéramos apartarnos de la ortografía española! [...] Poquito a poco esta efervescencia superficial pasará, y las generaciones futuras nos lo agradecerán. (Viana 1911)

Con esta operación retórica de cuestionar a España para criticar la ortografía de la RAE, Lenz reactiva la actitud romántica que se gestó en un grupo de letrados americanos a principios del siglo XIX, quienes se caracterizaban por su fuerte tendencia liberal y democrática en lo político, y un marcado, muchas veces inapelable, sentimiento antiespañol. Retoma el antiespañolismo que 
Domingo F. Sarmiento divulgó con tanto empeño, que buscaba romper los lazos culturales y nacionales que oprimían a las excolonias españolas. España representa entonces, lo que en términos sarmientinos es la barbarie que se opone a la civilización. Este recurso utilizado por Lenz puede ser interpretado en términos de la activación de una memoria discursiva (Arnoux 2008: 44) que se configura como una estrategia persuasiva para conseguir apoyo a la conservación de la ortografía chilena, asociando lo español al atraso y el retroceso, opuesto al progreso y la civilización.

Finalmente, cabe destacar que Lenz concede, en algún punto, que la RAE de "hoy" (última década del XIX) adolecía de todos estos problemas, pero no siempre lo había hecho. Por el contrario, en un momento anterior, había dado preeminencia al criterio de la pronunciación, haciendo así de su ortografía un sistema racional. Tan solo al dar importancia excesiva a la etimología extravió el buen camino por el que iba:

Poco a poco, procediendo con sabia moderacion, $[\ldots]$ hasta el siglo XVIII, nunca se fijó la Real Academia en la etimolojía de las palabras. [...] De esta manera, al cabo de cien años de trabajo sabio i razonado, a principios del siglo XIX, la ortografía de la Academia estaba cerca de llegar a la perfeccion ideal. (Lenz 1894b: 48)

\subsubsection{La tradición ortográfica chilena}

Otra forma estratégica en que Lenz se posiciona frente a la tradición es la construcción de una imagen de "continuidad" para la postura favorable a la ortografía reformada. Creemos que el propósito de esta estrategia es apelar al sentido común de los sectores conservadores, nuevamente, para quienes, se sabe, la tradición, como ideologema, podía ser un argumento muy atractivo, sobre todo si era una tradición propiamente chilena.

Lenz insiste en que una correcta consideración de la ortografía reformada, no en la década de 1840 sino que ya en la última década del siglo XIX, debe tener en cuenta que se trata "solo de conservar la ortografía acostumbrada de Chile" (Lenz 1894b: 26). Y agrega:

No se trata hoi de ninguna innovacion, de ninguna reforma nueva en materias ortográficas, sino esclusivamente de la cuestion si debemos conservar i prescribir definitivamente para todos los establecimientos de enseñanza del Estado i para todas las publicaciones oficiales, aquella ortografía que debe su orijen al jenio de un Andres Bello i que hasta el dia de hoy goza de la mas jeneral aceptacion en Chile, o si debemos abandonar las costumbres seguidas en los últimos diez lustros para aceptar la Ortografía que tiene a bien prescribir la Real Academia Española. (Lenz [1891] 1894a: 19) 
De acuerdo con Lenz, entonces, favorecer la ortografía chilena no era una postura rupturista, provocadora de caos, sino más bien conservadora, pues esta ortografía reformada ya era una tradición, y con ello un principio de orden y estabilidad (además ratificado por la voluntad de los sujetos escribientes, como sugiere lo de la "jeneral aceptacion"). No es casualidad que Lenz atraiga (como hace reiteradamente en estos textos) la figura de autoridad de Bello, a quien se remonta la ideología lingüística hegemónica de las élites hispanohablantes de este periodo, para reforzar la evocación de este significado indicial (la "tradicionalidad") para la ortografía reformada chilena.

Si el hecho de que el mismo Bello haya sido un partidario del reformismo es un refuerzo para la postura pro ortografía chilena, todavía de más peso puede ser para los interlocutores de Lenz el que el reformismo coincida en espíritu con lo que la propia RAE había venido haciendo desde comienzos del XIX, como vimos en 3.3.1. En el fondo, quiere decir Lenz, hasta la propia RAE fue reformista en su mejor época. Así, al destacar el vínculo de la postura reformista con el gramático venezolano, aprovecha de prolongar la filiación hacia la corporación española:

Hai que insistir en que las reformas de Bello, comenzadas desde 1823 por su estudio intitulado $<<$ Conveniencia de simplificar la Ortografía $>>$ [...] son la lejítima continuacion de la meritoria obra que la Real Academia Española habia comenzado en 1713, i continuado con vigor i sin miramientos para con la $<<$ novedad $>>$, hasta el año de 1815. (Lenz 1894b: 12)

Esperemos que la Academia siga cuánto ántes el modelo de Bello, como éste en 1823 siguió el modelo de la Academia de 1815. (Lenz 1894b: 18)

En suma, tal como lo describe Lenz de una forma que pudiera parecer oximorónica, lo que debía hacerse en Chile era "conservar los progresos". (Lenz [1891] 1894a: 54, 59)

Dos ideologemas más aparecen articulados con la construcción de una imagen "tradicional" para la ortografía reformada chilena: el del gradualismo y el de la unidad, ambos de clara filiación bellista (Jaksic 2015).

El gradualismo apuntaba a que todo cambio social se diera de manera progresiva y bajo los sustentos firmes de la tradición. Para aquellos intelectuales que participaron en los procesos independentistas era fundamental que, en terreno político, las cosas fuesen cambiando, pero no de forma abrupta y radical, sino mediante procesos graduales que permitiesen una adaptación paulatina. Así, por ejemplo, el tránsito del orden monárquico al orden republicano idealmente debía ser gradual, en el sentido de que se 
mantuviesen algunas instituciones culturales coloniales, a las cuales luego se les fuesen superponiendo las nuevas instituciones de la independencia. Y en terrenos de la lengua, algunos intelectuales -Andrés Bello dentro de estos- manifiestan esta idea de lo gradual mediante el "purismo moderado" (tal como queda explicado en el prólogo de la Gramática castellana que Bello publicó en 1847) que implicaba atender a los patrones culturales que regían y limitaban la lengua, pero sin con ello impedir que la lengua se fuese adaptando a los nuevos tiempos, dado que justamente la idea del progreso exigía que esta fuese cambiando.

En consecuencia, Lenz reitera la idea de que el reformismo no implicaba transformación radical ni nada por el estilo:

En materia de ortografía propiamente dicha, seria conveniente conservar por ahora lo que tenemos, i mas tarde continuar lentamente en el camino una vez comenzado, suprimiendo las pocas dificultades superfluas que todavía hai sin necesidad alguna. (Lenz 1894b: 59)

Por eso, no hai ninguna necesidad urjente para apresurar la marcha de la reforma. La razón vencerá, i ella está en el camino que pasa de la escritura academica por la de Bello a la del señor Newman. No nos apresuremos demasiado, pero no vayamos atras. (Lenz 1894b: 18)

Con la idea de la unidad, apela a quizá la idea de mayor peso en la ideología lingüística bellista (Rojas 2017). La homogeneidad, la estandarización de la lengua española, fue sentida por las élites gobernantes chilenas del temprano XIX como una necesidad para el funcionamiento apropiado de la nueva nación independiente. De tal manera, la percepción de la amenaza de la fragmentación conllevaba preguntarse qué medidas podían o debían tomarse para evitarla y quiénes debían estar a cargo de ellas. El tema principal, la obsesión, podría decirse, para estos sujetos, fue la unidad del idioma. En el contexto chileno, es Andrés Bello el primero que puso énfasis en el valor comunicativo y político de la unidad lingüística. En una declaración muy citada del prólogo de su Gramática de 1847, Bello expresó que promover un estándar, en torno al cual se aseguraría la unidad de la lengua española, permitiría contar con "un medio providencial de comunicación y un vínculo de fraternidad entre las varias naciones de origen español derramadas sobre los dos continentes" (Bello [1847] 2013: 42). Esta idea de la unidad -panhispánica-representaba la mayor preocupación de quienes se mostraban reacios a abandonar la ortografía de la RAE por la ortografía chilena.

Lenz creía que la unidad, de hecho, era una meta deseable; sin duda, creía en los beneficios de estandarizar el idioma (Soto 2016). Sin embargo, pensaba que la unidad no debía articularse sobre la ortografía de la RAE, 
sino sobre la ortografía reformada chilena, por tener esta las características deseables para la escritura (cientificidad, simplicidad, etc.):

Es verdad que se ha dicho que Chile no tiene el derecho de apartarse del uso comun de todos los pueblos hispanos, sancionado por la Real Academia Española. Pero tambien es verdad que la autoridad de la Academia no ha dejado de encontrar oposicion hasta en la misma España. (Lenz 1894b: 38)

[...] seria ventajosa i deseable la uniformidad de la ortografía en todos 1os paises de habla castellana, opino que seria mui doloroso dejar de mano mejoras usadas en Chile desde tres cuartos de siglo. (Lenz 1894b: 12)

Y esto a pesar de que se muestra escéptico de la posibilidad de que realmente se alcance esa unidad:

Esta deseable uniformidad absoluta de la ortografía, hasta ahora no existe de hecho en ningun país, en ninguna lengua, aunque hai entre casi todas las naciones cultas ciertos cánones sancionados por el uso, de los cuales nadie se puede apartar en mas que algunos puntos, jeneralmente de poca importancia. (Lenz [1891] 1894a: 41)

La unidad absoluta en cuestiones de ortografía castellana no ha existido nunca en América. (Lenz [1891] 1894a: 51)

En síntesis, el hecho de que Lenz introduzca la idea de la unidad en sus discursos sobre la ortografía reformada chilena, creemos, hay que entenderlo igualmente como parte de sus estrategias para persuadir a la élite hispanohablante chilena de la conveniencia de mantener la reforma, hablándoles en términos que les pudieran parecer atractivos.

\section{CONCLUSIONES}

La postura de Lenz, favorable sin duda a la ortografía reformada chilena y extremadamente crítica de la ortografía de la RAE, puede explicarse desde un enfoque glotopolítico atendiendo a los elementos contextuales que inciden y se manifiestan en la construcción discursiva de representaciones ideológicas de los modelos ortográficos en pugna en Chile en la última década del siglo XIX. En esta representación ideológica, o ideología lingüística, los modelos ortográficos en cuestión son cargados de elementos valorativos que se pueden 
sintetizar en los siguientes significados indiciales (Silverstein 2003), de los cuales los de la columna izquierda tienen polaridad positiva, mientras que los de la derecha tienen polaridad negativa, para Lenz:

\begin{tabular}{|c|c|}
\hline Ortografía reformada chilena & Ortografía de la RAE \\
\hline Cientificidad & Acientificidad \\
\hline Razón & Irracionalidad \\
\hline Progreso & Retraso \\
\hline Tradición (chilena, propia) & Tradición (española, ajena) \\
\hline Orden & Caos \\
\hline Simplicidad (aplicación práctica) & Complejidad (en aprendizaje) \\
\hline
\end{tabular}

Tabla 1. Significados indiciales de los modelos ortográficos en pugna en Chile por la década de 1890, según Lenz

En cuanto a los elementos contextuales, en primer lugar, hay que considerar que Lenz, como sujeto político, tiene un rol hipercentral en la creación de un campo científico moderno en Chile en lo que atañe al estudio de las lenguas y manifestaciones culturales asociadas (folklore, literatura popular, etc.), de modo que no podía sino opinar como un científico, "encarnando" o asumiendo la voz de la ciencia moderna, por así decirlo. El eje discursivo de "lo científico" activa, al mismo tiempo, otros conceptos clave para persuadir a los gobernantes de la conveniencia de mantener vigente la ortografía reformada: la razón y el progreso. En esto, Lenz responde a las expectativas que se tenían de él de acuerdo con los planes del gobierno de modernizar en general el Estado y la creación y transmisión de conocimientos en el país. El fin de siglo chileno, comenzando por 1880, está marcado por el crecimiento económico y por el consecuente afán de modernización del país (Subercaseaux 2011), y por "modernizar" se entendía el hacer a Chile semejante a otras naciones que se percibían como "modernas". Por ello, Lenz abre la discusión a la comparación con otros países, teniendo en cuenta su estatus científico, de tal modo que España, asociada a la ortografía académica, queda mal parada, deslegitimando así los fundamentos de la norma de la RAE. En última instancia, esta estrategia discursiva de Lenz en los debates sobre la ortografía forma parte de su estrategia mayor de posicionamiento y autolegitimación en el medio local, que desde un principio le fue adverso intelectualmente.

En segundo lugar, Lenz construye su argumento teniendo muy en cuenta que sus interlocutores, los sujetos capaces de tomar o promover una decisión política respecto de la pugna ortográfica (pensemos, por ejemplo, en un 
Miguel Luis Amunátegui Reyes), forman parte de una élite hispanohablante todavía fuertemente marcada por la ideología lingüística hegemónica en Chile desde comienzos del XIX, inspirada fundamentalmente en las ideas de Andrés Bello. De tal modo, construye para la ortografía reformada una representación en que, al contrario de lo que pudiera pensarse, esta no es innovadora sino tradicional, y todavía más digna de preservarse por ser una tradición propiamente chilena y que trajo progreso a la nación. Al presentarse como tradicional, aparece afiliada al mismo Bello e incluso a la propia $\mathrm{RAE}$, en un momento en que esta seguía criterios racionales y científicos. Al ser tradición, la ortografía reformada chilena posibilita el cambio gradual dentro de un orden, que es quizá el ansia más grande de las élites gobernantes chilenas incluso en las décadas finales del siglo XIX (Stuven 2000). Más allá de los límites de Chile, el orden y racionalidad de la ortografía chilena posibilitaría incluso la unidad ortográfica de todas las naciones de habla castellana.

Con su intervención en las polémicas sobre la ortografía reformada chilena, Lenz marcaría una senda e inspiraría a futuros intervinientes que también adoptaron una postura similar, a veces radicalizándola, como en el caso de los neógrafos (que no por nada editaron en 1914 reunidos en un solo tomo los dos textos principales analizados en el presente estudio), o a veces siguiéndola de manera más fiel, como en el caso de Miguel Luis Amunátegui Reyes, quien, por el lugar hipercentral que años más tarde ocuparía en la Academia Chilena de la Lengua, serviría de amplificador para la opinión prorreforma. Esto demuestra que, a pesar de que la postura de Lenz fue contrahegemónica en relación con el campo cultural chileno, y de que finalmente la cuestión se resolvió en favor de la ortografía de la RAE, sus ideas ortográficas lograron persuadir a un sector no menos importante de la intelectualidad chilena de fines del XIX y comienzos del XX.

Al corresponder a una de sus primeras intervenciones en los debates públicos sobre las lenguas en Chile, los textos examinados acá muestran, por un lado, lo temprano del compromiso de Lenz con el ejercicio de su rol de intelectual público en el contexto finisecular de Chile, marcado por importantes transformaciones sociales, culturales, económicas y políticas; recuérdese que el primer texto de la serie es de 1891, es decir, fue publicado a tan solo un año de haber llegado a Chile. Por otro lado, revelan que desde ese primer momento el filólogo alemán ya estaba activando categorías e ideas que articularán más adelante su postura frente a diversos problemas lingüísticos, tales como el estudio de las lenguas indígenas, del castellano chileno y la enseñanza de segundas lenguas. Estas ideas, moldeadas por el cientificismo positivista, específicamente bajo la forma de la lingüística histórico-comparada de los neogramáticos, al mismo tiempo exhiben una 
aguda conciencia, por parte de Lenz, de enfrentarse a problemas no solo lingüísticos, sino genuinamente glotopolíticos, es decir, problemas en que lo lingüístico y lo político se dan de forma indisociable.

\section{REFERENCIAS BIBLIOGRÁFICAS}

\section{Fuentes primarias}

1.1. Obras publicadas

Lenz, Rodolfo. [1891] 1894a. Observaciones sobre la ortografía castellana. Anales de la Universidad de Chile LXXXVII: 570-579. 559-569 1894b. De la ortografía castellana. Anales de la Universidad de Chile LXXXVII: 1914. De la ortografia castellana. Segunda edizion. Balparaíso: Franzisko Enrríkez.

1.2. Materiales inéditos

Barros Grez, Daniel. 1896(a). Carta dirigida a Rodolfo Lenz, 30 de abril de 1896. Quillota. 1896(b). Carta dirigida a Rodolfo Lenz, 18 de mayo de 1896, Quillota. 1896(c). Carta dirigida a Rodolfo Lenz, 12 octubre 1896, Quillota.

Echeverría y Reyes, Aníbal. 1894a. Carta dirigida a Rodolfo Lenz, 29 de septiembre 1894, Valparaíso. 1894b. Carta dirigida a Rodolfo Lenz, 7 de noviembre 1894, Valparaíso. 1894c. Carta dirigida a Rodolfo Lenz, 4 de X 1894, Valparaíso. 1895. Carta dirigida a Rodolfo Lenz, 6 enero 1895, Valparaíso.

Lenz, Rodolfo. s. f. Borrador de carta dirigida a Miguel Luis Amunátegui Reyes, Santiago. Viana, Aniceto do Reis Gonçalves. 1896. Carta dirigida a Rodolfo Lenz, 14 de agosto de 1896. Lisboa. 1911. Carta dirigida a Rodolfo Lenz, 29 septiembre 1911. Lisboa.

\section{Bibliografía secundaria}

Álvarez Martínez, María Ángeles. 1997. Rodolfo Lenz: contribución gramatical y lexicográfica. Historiographia Lingüística 24 (1-2): 197-212.

Arnoux, Elvira. 2006. Análisis del discurso. Modos de abordar materiales de archivo. Buenos Aires: Santiago Arcos.

2008. Los discursos sobre la nación y el lenguaje en la formación del Estado (Chile, 1842-1862). Estudio glotopolitico. Buenos Aires: Santiago Arcos.

y del VAlLe, José (eds.). 2010. Las representaciones ideológicas del lenguaje. Discurso glotopolítico y panhispanismo. Spanish in Context 7(1): 1-24.

Bello, Andrés. [1847] 2013. Prólogo de Gramática de la lengua castellana destinada al uso de los americanos. En Andrés Bello, Gramática de la libertad. Textos sobre lengua y literatura, comps. I. Jaksic, F. Lolas y A. Matus, pp. 43-50. Santiago: Universidad de Chile.

BernasChinA, Vicente. 2013. Rodolfo Lenz, 150 años en disputa con el imperio de la Lengua. Universum 28(2): 117-138. 
2016. Rodolfo Lenz: hacia una filología crítica americana. En Sergio Ugalde y Ottmar Ette (eds.), Politicas y estrategias de la crítica: ideología, historia y actores de los estudios literarios, pp. 119-137. Madrid/Frankfurt: Iberoamericana/Vervuert.

Chávez, Soledad. 2011. Ideas lingüísticas de Lenz en los paratextos de su diccionario etimológico de las voces chilenas derivadas de lenguas indígenas americanas: vigencia y urgencia en el español de Chile. Lenguas Modernas 38(2): 83-106.

Contreras, Lidia. 1993. Historia de las ideas ortográficas en Chile. Santiago: Centro de Investigaciones Diego Barros Arana.

1994. Las ideas ortográficas de Rodolfo Lenz. En Ortografía y grafémica, pp. 49-60. Madrid: Visor.

Del Valle, José. 2016. Lenguaje, política e historia: ensayo introductorio. En José del Valle (ed.). Historia política del español: la creación de una lengua, pp. 3-26. Madrid: Aluvión. 1: $17-39$.

2017. La perspectiva glotopolítica y la normatividad. Anuario de Glotopolítica

Y Vitor MeIRINHo-Guede. 2016. Ideologías lingüísticas. En Javier Gutiérrez-Rexach (ed.). Enciclopedia de lingüística hispánica, vol. 2, pp. 622-631. London \& New York: Routledge.

EnNis, JuAn Antonio. 2012. Rudolf Lenz en la encrucijada criolla. Signo y Seña 22: 181-214. 2014. August Schleicher: los dos cuerpos de la lengua. Revista Argentina de Historiografia Lingüistica 6(2): 107-121.

2016. Rodolfo Lenz: economías de la lengua y políticas de la lingüística. Boletín de Filología 51(1). 117-145.

Escudero, Alfonso M. 1963. Rodolfo Lenz. Thesaurus 18(2): 445-484.

García del Río, JuAn y ANdrés Bello. ([1823] 2013). Indicaciones sobre la conveniencia de simplificar i uniformar la ortografía en América. En Andrés Bello, Gramática de la libertad. Textos sobre lengua y literatura, comps. I. Jaksic, F. Lolas y A. Matus, pp. 4558. Santiago: Universidad de Chile.

Gómez Asencio, José Jesús. 2016. Categorías en La oración y sus partes (1920) de Rodolfo Lenz: Clases. Boletín de Filología 51(1): 147-185.

GutiÉRREZ, DANIELA. (2017). Reforma ortográfica e ideologías lingüisticas en Chile (1913 1927): la Academia Chilena de la Lengua y el retorno a la ortografia de la RAE. Tesis de magíster, Universidad de Chile.

JAKSIĆ, IVÁN. 1999. La gramática de la emancipación. En Germán Carrera Damas y John Lombardi (dirs.). Historia general de América Latina, vol. 5, pp. 507-521. Madrid: Unesco/Trotta.

2015. Los Cuadernos de Londres de Andrés Bello. Boletín de Filología 50(2): 181-189.

KNAUER, GABRiele. 1993. Filólogos alemanes en América Latina: Rodolfo Lenz y el europeísmo cultural en el discurso científico de la lingüística iberoamericana. En Hans-Otto Dill y Grabriele Knauer (eds.). Diálogo y conflicto de culturas: Estudios comparativos de procesos transculturales entre Europa y América Latina, pp. 139-152. Frankfurt: Vervuert. y Martin Kaluza. 1998. Die 'indigenistische Theorie' der Phonetik im chilenischen Spanisch von Rodolfo Lenz: Ein Vorläufer der modernen Kontaktlinguistik? Philologie im Netz 3: 1-21

Koerner, E. F. K. 2008. Hermann Paul and general linguistic theory. LanguageSciences 30: $102-132$.

Larraín, Jorge. 2007-2010. El concepto de ideología. 4 vols. Santiago: Lom.

Lenz, Rodolfo. s.f. Anexo segundo al Diccionario etimológico. Santiago: Edición no venal del autor. 
MarTínez AlCAlde, María José. 2010. La fijación ortográfica del español: norma y argumento historiográfico. Bern: Peter Lang.

Martínez Celdrán, Eugenio, y Lourdes Romera Barrios. 2007. Historiografía de la fonética y fonología españolas. En Josefa Dorta, Cristóbal Corrales y Dolores Corbella (eds.), Historiografía de la lingüistica en el ámbito hispánico. Fundamentos epistemológicos y metodológicos, pp. 119 - 160. Madrid: Arco Libros.

Martínez, José Antonio. 1997. El funcionalismo de Rodolfo Lenz. Una tradición de América a España. HistoriographiaLingüistica 24(3): 307-330.

MuÑz CACHÓn, CARmen. 2009. Fernando de Araujo en los orígenes de la fonética hispánica. En José María García Martín y Victoriano Gaviño Rodríguez (eds.), Ideas y realidades lingüisticas en los siglos XVIII y XIX, pp. 483-497. Cádiz: Universidad de Cádiz.

Ortiz LiRa, HÉctor. 1994. A hundred years of the English Department. English Review 1: 3-35.

Pavez, Jorge. 2015. La lengua de Chile: Rodolfo Lenz entre chilenos y mapuches. En Laboratorios etnográficos. Los archivos de la antropología en Chile (1880-1980), pp. 67-167. Santiago: Ediciones Universidad Alberto Hurtado.

PayÁs, GeTRUdis. 2008. Tradukzión i rrebeliónortográfika. Trans. Revista de Traductología 12: $15-28$.

2015. Tan verídica como patriota: La pugna sobre traducción entre Rodolfo Lenz y Manuel Manquilef. CUHSO 25(2): 83-114.

Rojas, Darío. 2011. Voces usadas en Chile (1900): las cartas de Aníbal Echeverría y Reyes a Rodolfo Lenz. Onomazein 24: 349-361

2015. Ideologías y actitudes lingüisticas en el Chile hispanohablante de la segunda mitad del siglo XIX. Tesis doctoral, Universidad de Valladolid

2016. The Chilean Academy of the Spanish Language: the institutionalization of a discourse community. History and Philosophy of the Language Sciences, https:// hiphilangsci.net

2017. Representaciones del cambio lingüístico en Chile durante el siglo XIX: ‘¿progreso o decadencia?’. Literatura y Lingüística 36: 243-262.

2018. La reforma ortográfica y la Academia Chilena. Las actas de la Academia Chilena de la Lengua: institucionalidad, lengua y literatura en Chile (1885-1993), pp. 37-38. Santiago, edición no venal, www.actasachl.cl

SÁnchez, Gilberto. 2013. El Dr. Rodolfo Lenz, primer investigador científico de la lengua y cultura mapuches. Lenguas Modernas 42(2): 99-113.

Sanhueza, Carlos. 2010. Circulación de intelectuales alemanes en Chile y chilenos en Alemania (fines del siglo XIX y comienzos del siglo XX). História: Questoes \& Debates 53: $67-84$.

Silverstein, Michael. 2003. Indexical order and the dialectics of sociolinguistic life. Language and Communication 23: 193-229.

Soto, Guillermo. 2016. Rodolfo Lenz y la enseñanza del castellano como idioma patrio en Chile. Boletín de Filología 51(1): 211-238.

Stuven, Ana María. (2000). La seducción de un orden. Las elites y la construcción de Chile en las polémicas culturales y políticas del siglo XIX. Santiago: Ediciones de la Universidad Católica de Chile.

Subercaseaux, Bernardo. 2011. Historia de las ideas y la cultura en Chile: desde la Independencia hasta el Bicentenario. Tomo 1, volumen 1: Sociedad y cultura liberal en el siglo XIX. Santiago: Editorial Universitaria.

Swiggers, Pierre. (2011). 19th century linguistics: practice and theory. En Bernd Kortmann y Johan van der Auwera (eds.). The languages and linguistics of Europe: a comprehensive guide, pp. 805-820. Berlin/Boston: Walter de Gruyter. 
VAn der Hulst, Harry. 2013. Discoverers of the phoneme. En Keith Allan (ed.). The Oxford handbook of the History of Linguistics, pp. 167-191. Oxford: OUP.

Velleman, BarRy. 2004. Antiacademicismo lingüístico y comunidad hispánica: Sarmiento y Unamuno. En José del Valle y Luis Gabriel-Stheeman (eds.). La batalla del idioma: la intelectualidad hispánica ante la lengua, pp. 35-65. Madrid/Frankfurt: Iberoamericana/ Vervuert.

2007. Las ideas lingüísticas y pedagógicas de Eduardo de la Barra y su polémica con Lenz y Hanssen. Contextos 18: 179-193.

2008. La imagen y los ecos del lingüista profesional: la correspondencia de Rodolfo Lenz. RLA. Revista de Lingüística Teórica y Aplicada 46(1): 11-28.

VILLA, LAURA. 2016. La oficialización del español en el siglo XIX. La autoridad de la Academia. En José del Valle (ed.). Historia política del español: la creación de una lengua, pp. 107-121. Madrid: Aluvión.

Villa, Laura, y Rik Vosters. 2015. Language ideological debates over orthography in European linguistic history. Written Language \& Literacy 18(2): 201-207.

Villarroel, Natalia. 2017. Reforma ortográfica y comunidad discursiva: los neógrafos chilenos o "los intrépidos revolucionarios del abecé" (1892-1927). Tesis de magíster, Universidad de Chile. 